\title{
Mapping and validation of QTLs for rice sheath blight resistance
}

\author{
Fumio Taguchi-Shiobara*1), Hidenobu Ozaki ${ }^{2,4)}$, Hiroyuki Sato ${ }^{3,5)}$, Hiroaki Maeda ${ }^{2,6)}$, Yoichiro Kojima ${ }^{2,7)}$, \\ Takeshi Ebitani' ${ }^{2}$ and Masahiro Yano1) \\ 1) National Institute of Agrobiological Sciences, 2-1-2 Kannondai, Tsukuba, Ibaraki 305-8602, Japan \\ 2) Toyama Prefectural Agricultural, Forestry \& Fisheries Research Center, 1124-1 Yoshioka, Toyama, Toyama 939-8153, Japan \\ 3) National Agriculture and Food Research Organization (NARO) Institute of Crop Science, 2-1-18 Kannondai, Tsukuba, Ibaraki 305- \\ 8518, Japan \\ 4) Present address: Agricultural Food Product Division, Toyama Prefecture, 1-7 Shin Sougawa, Toyama, Toyama 930-8501, Japan \\ 5) Present address: NARO Kyushu Okinawa Agricultural Research Center, 496 Izumi, Chikugo, Fukuoka 833-0041, Japan \\ 6) Present address: Takaoka Agricultural Forestry Promotion Center, 211 Akasofu, Takaoka, Toyama 933-0806, Japan \\ 7) Present address: Agricultural Technology Division Regional Extention Center, 1124-1 Yoshioka, Toyama, Toyama 939-8153, Japan
}

Sheath blight, caused by Rhizoctonia solani, is one of the most serious diseases of rice. Among 33 rice accessions, mainly from National Institute of Agrobiological Sciences (NIAS) Core Collection, we found three landraces from the Himalayas-Jarjan, Nepal 555 and Nepal 8-with resistance to sheath blight in 3 years' field testing. Backcrossed inbred lines (BILs) derived from a cross between Jarjan and the leading Japanese cultivar Koshihikari were used in QTL analyses. Since later-heading lines show fewer lesions, we used only earlier-heading BILs to avoid association with heading date. We detected eight QTLs; the Jarjan allele of three of these increased resistance. Only one QTL, on chromosome 9 (between markers Nag08KK18184 and Nag08KK18871), was detected in all 3 years. Chromosome segment substitution lines (CSSLs) carrying it showed resistance in field tests. Thirty $\mathrm{F}_{2}$ lines derived from a cross between Koshihikari and one CSSL supported the QTL.

Key Words: rice sheath blight resistance, Oryza sativa L., QTL, chromosomal segment substitution line, CSSL, Rhizoctonia solani.

\section{Introduction}

Sheath blight, caused by Rhizoctonia solani, is a major disease of rice, second in importance only to rice blast, in many part of the world (Rush and Lee 1992). This soilborne pathogen has a broad host range and a worldwide distribution and infects plants in more than 32 families and 188 genera (Gangopadyay and Chakrabarti 1982). Sheath blight causes severe losses in yield and grain quality in many rice-growing countries in both tropical and temperate climates (Banniza and Holderness 2001). It is aggravated by warm and humid weather, dense planting and high nitrogen inputs (Hashiba and Kobayashi 1996).

Rice genetic resources have not been comprehensively exploited for improvement of sheath blight resistance, although many cultivars and lines have been reported as promising sources of resistance (Srinivasachary et al. 2011). In producing hybrid populations for QTL analysis, several sources have been used, including Teqing (Li et al. 1995, Pinson et al. 2005), Jasmine 85 (Liu et al. 2009, Pan et al. 1999, Zou

Communicated by H. Kato

Received March 25, 2013. Accepted May 4, 2013.

*Corresponding author (e-mail: fstagu@affrc.go.jp) et al. 2000), Zhai Ye Qing 8 (Kunihiro et al. 2002), Minghui 63 (Han et al. 2002), Xiangzaoxian 19 (Che et al. 2003), WSS2 (Sato et al. 2004), Tetep (Channamallikarjuna et al. 2010) and Pecos (Sharma et al. 2009). Some have been used as donors to develop near-isogenic lines (NILs) or chromosome segment substitution lines (CSSLs) to identify QTLs that can measurably improve resistance. Yin et al. (2009) showed that a 3.9-Mb Teqing fragment between markers RM242 and Y92.5 in the $q S B-9$ (QTL for sheath blight resistance on chromosome 9) region in the Lemont background increased resistance. Wang et al. (2011) located $q S B 9-2$ in a $1.8 \mathrm{-Mb}$ region (Rice Annotation Project et al. 2008) between RM245 and the end of chromosome 9 and qSB12-1 in a 7.9-Mb region on chromosome 12 .

The development of sheath blight lesions reflects not only plant genotype, but also temperature and humidity, which are affected by plant density or tiller number (Hashiba and Kobayashi 1996) as well as climate. Early-heading cultivars appear more susceptible than later-heading ones, because conditions in late summer become less favorable for sheath blight development. QTLs for sheath blight resistance have been often detected in the same region as QTLs for heading date (Li et al. 1995, Pinson et al. 2005, Sharma et al. 2009): when cultivars of different maturation times 
were planted so as to head at the same time, the differences in resistance became smaller (Lee and Rush 1983). They have also been detected in the same region as QTLs for plant height (Li et al. 1995, Pinson et al. 2005, Sharma et al. 2009, Zou et al. 2000): when the ratio of the height of the highest lesion to plant height is used in evaluating resistance taller plants tend to be scored as more resistant.

To identify new sources of resistance to sheath blight which could be applied to improving cultivars, we screened accessions and identified three resistant landraces from the Himalayas: Jarjan, Nepal 555 and Nepal 8. A QTL analysis using backcrossed inbred lines (BILs) from a cross between Jarjan (J) and Koshihikari (K) detected the same QTL in each of 3 years. The effect of this QTL was verified by field tests of CSSLs.

\section{Materials and Methods}

\section{Plant materials}

We screened 33 rice accessions for resistance to sheath blight (Table 1), 27 from the NIAS Core Collection conserved in the NIAS Genebank (Ebana et al. 2008, Kojima et al. 2005; http://www.gene.affrc.go.jp/databasescore_collections_en.php) and 6 others, including the leading Japanese cultivar, Koshihikari, the resistant cultivar WSS3, derived from Tetep (Ohuchida et al. 2008, Wasano 1988) and the susceptible cultivar Lemont. These accessions were sown in late March and transplanted on 23-25 April in the field at Toyama Prefectural Agricultural, Forestry and Fisheries Research Center, Toyama, in 2008, 2009 and 2010. Every year 15 plants of each accession with 2 replications were evaluated. In addition, we grew 95 BILs $\left(\mathrm{BC}_{1} \mathrm{~F}_{5}: \mathrm{J} / \mathrm{K} / / \mathrm{K}\right.$; Abe et al. 2011, Taguchi-Shiobara et al. 2011; http://www.rgrc.dna.affrc.go.jp/ineJKKBIL95.html)

Table 1. List of 33 accessions used in 3 years' field testing for resistance to sheath blight

\begin{tabular}{|c|c|c|c|c|}
\hline Accession Name & $\mathrm{ID}^{a}$ & Origin (Country) & Origin (Area) & Subspecies \\
\hline NIPPONBARE & WRC 01 & Japan & - & japonica \\
\hline PULUIK ARANG & WRC 06 & Indonesia & - & indica \\
\hline $\mathrm{ASU}$ & WRC 13 & Bhutan & - & indica \\
\hline $\mathrm{CO} 13$ & WRC 15 & India & - & indica \\
\hline SHWE NANG GYI & WRC 21 & Myanmar (Burma) & - & indica \\
\hline MUHA & WRC 25 & India & - & indica \\
\hline NEPAL 8 & WRC 27 & Nepal & - & indica \\
\hline JARJAN & WRC 28 & Bhutan & - & indica \\
\hline ANJANA DHAN & WRC 30 & Nepal & - & indica \\
\hline NEPAL 555 & WRC 40 & India & - & indica \\
\hline KALUHEENATI & WRC 41 & Srilanka & - & indica \\
\hline PADI PERAK & WRC 49 & Indonesia & - & tropical japonica \\
\hline URASAN 1 & WRC 51 & Japan & - & japonica \\
\hline KHAU TAN CHIEM & WRC 52 & Vietnam & - & japonica \\
\hline DEEJIAOHUALUO & WRC 98 & China & - & indica \\
\hline HONG CHEUH ZAI & WRC 99 & China & - & indica \\
\hline SENSHOU & JRC 04 & Japan & Tokyo & tropical japonica \\
\hline KANEKO B & JRC 06 & Japan & Kantou Touzan & tropical japonica \\
\hline WATARIBUNE & JRC 19 & Japan & Shiga & japonica \\
\hline MANSAKU & JRC 22 & Japan & Nagano & japonica \\
\hline SHINRIKI MOCHI & JRC 28 & Japan & Kumamoto & japonica \\
\hline SHICHIMENCHOU MOCHI & JRC 29 & Japan & (unknown) & japonica \\
\hline SHINYAMADAHO 2 & JRC 37 & Japan & Hyougo & japonica \\
\hline NAGOYA SHIRO & JRC 38 & Japan & Akita & japonica \\
\hline SHIROINE(KEMOMI) & JRC 39 & Japan & Tokushima & japonica \\
\hline AKAMAI & JRC 40 & Japan & Nagasaki & indica \\
\hline KARAHOUSHI & JRC 44 & Japan & Kagoshima & indica \\
\hline KOSHIHIKARI & - & Japan & Toyama $^{b}$ & japonica \\
\hline SASANISHIKI & - & Japan & (unknown) & japonica \\
\hline TENTAKAKU & - & Japan & Toyama $^{b}$ & japonica \\
\hline LEMONT & - & USA & - & - \\
\hline CHUUGOKU 117 & - & Japan & Hiroshima & - \\
\hline WSS3 & - & Japan & Kagoshima $^{c}$ & - \\
\hline
\end{tabular}

${ }^{a}$ ID of NIAS Core Collection. WRC, Global Core Collection; JRC, Core Collection of Japanese Landraces.

${ }^{b}$ Distributed by Toyama Prefectural Agricultural, Forestry and Fisheries Research Center.

${ }^{c}$ Distributed by Kagoshima Prefectural Institute for Agricultural Development. 
to detect QTLs from 2009 to 2011.

We crossed Koshihikari with Jarjan and backcrossed a resultant $F_{1}$ plant with Koshihikari to produce $22 \mathrm{BC}_{1} \mathrm{~F}_{1}$ plants. Marker-assisted selection showed one of these plants to have heterologous alleles at the DNA marker loci RM5122 and RM6971 on chromosome 9 (near a candidate QTL for sheath blight resistance; see Results) and we backcrossed this $\mathrm{BC}_{1} \mathrm{~F}_{1}$ with Koshihikari to obtain $\mathrm{BC}_{2} \mathrm{~F}_{1}$ plants. Two generations of selfing and marker-assisted selection using 152 simple sequence repeat (SSR) markers resulted in five $\mathrm{BC}_{2} \mathrm{~F}_{3}$ plants, which we selected as CSSLs with Jarjan alleles at six markers on chromosome 9 which fully or partially covered the region of the QTL for sheath blight resistance (for convenience denoted as $q S B R-9$ ). These five CSSLs, qM1 and qM3-qM6, were evaluated in the field in Toyama in 2010 and 2011.

One CSSL, qM1, was used for single-nucleotide polymorphism (SNP) typing by 768 SNPs (Ebana et al. 2010) and was evaluated in the field at the Yawara Lowland Experimental Station of the National Institute of Crop Science, Tsukuba, in 2010. This CSSL was crossed with Koshihikari to produce $\mathrm{BC}_{3} \mathrm{~F}_{1}$ plants. From those plants, we selected 15 with the Jarjan allele at $q S B R-9$ and a further 15 with the Koshihikari allele. These 30 plants were selfed to obtain $\mathrm{BC}_{3} \mathrm{~F}_{2}$ lines, which were evaluated in the field at Toyama in 2011.

\section{Evaluation of resistance to sheath blight}

Two Rhizoctonia solani isolates, CS-2 and 90W-14, were used. CS-2 was held at Tsukuba and 90W-14, isolated from the field in Aichi Prefecture, was held at Toyama and Tsukuba (Supplemental Table 1). Each was stored on barley grains at $-20^{\circ} \mathrm{C}$ (Gaskill 1968, Naito et al. 1993).

Two methods of inoculation were used for field tests (Supplemental Table 1). The main method used was inoculation of soil with infected hulls. The pathogen was precultured for a few days on potato dextrose agar (PDA) medium at $28^{\circ} \mathrm{C}$. Mycelium was then cultured on PDA medium for 14 days at $28^{\circ} \mathrm{C}$ and then subcultured for another 14 days. A disc of medium with mycelium was added to an autoclaved mixture of $75 \mathrm{~g}$ rice hulls, $75 \mathrm{~g}$ bran and $150 \mathrm{~mL}$ polypeptone solution $(1 \% \mathrm{w} / \mathrm{v})$, and the mixture was incubated at room temperature. After 21 days, this infected mixture was mixed with $300 \mathrm{~g}$ of rice hulls and sprinkled by hand over four rows consist of 400 plants (each row representing a single plot with 15 plants per line, $25 \mathrm{~cm}$ between rows and $12.5 \mathrm{~cm}$ between plants). All rows were inoculated three times at intervals of about 9 days in late June and early July under flooded condition, in the daytime without wind or rainfall. Only 10 or 15 plants from the middle of a row were evaluated. We scored the proportion of tillers with lesions per total number of tillers in a plant and calculated the mean for each cultivar or line. We scored the ratio of the highest lesion height to plant height as relative lesion height (\%).

We also inoculated plants by syringe (Sato et al. 2004, Wasano et al. 1983). Mycelium was cultured in potato sucrose agar medium containing $1 \%(\mathrm{w} / \mathrm{v})$ polypeptone. Homogenized medium containing mycelium was injected into the third leaf sheath below the flag leaf at the heading date. At 28 days after inoculation, we scored the ratio of lesion area to total leaf sheath area of the second leaf. Three leaf sheaths per plant were inoculated and the mean score was calculated.

\section{QTL analysis and statistical analysis}

Linkage maps were constructed from the genotype data in MAPMAKER/EXP 3.0 software (Lander et al. 1987). The genetic distance was estimated by using the software's Kosambi map function. QTL analyses were performed by composite interval mapping as implemented by QTL Cartographer 2.5 software (http://statgen.ncsu.edu/qtlcart/ WQTLCart.htm). Genome-wide threshold values $(\alpha=0.05)$ were used to detect putative QTLs on the basis of the results of 1000 permutations (Churchill and Doerge 1994).

To compare $\mathrm{BC}_{2} \mathrm{~F}_{3}(\mathrm{~J} / \mathrm{K} / / \mathrm{K}), \mathrm{F}_{1}(\mathrm{~K} / \mathrm{qM} 1)$, or $\mathrm{BC}_{3} \mathrm{~F}_{2}$ lines (J or K alleles) with other lines or Koshihikari growing in the same field and to compare the $\mathrm{BC}_{3} \mathrm{~F}_{2}$ lines ( $\mathrm{J}$ or $\mathrm{K}$ alleles) with each other, we used the $F$-test and $t$-test provided in Microsoft Excel 2007. To compare panicle number among the four $\mathrm{BC}_{2} \mathrm{~F}_{3}$ lines (qM3-qM6) and Koshihikari, we used Tukey's multiple comparison test in JMP v. 10 software (SAS Institute, Cary, NC, USA).

The locations of markers were based on the sequence of the International Rice Genome Sequencing Project (IRGSP) Build 5 Pseudomolecules of the Rice Genome (Rice Annotation Project et al. 2008).

\section{Results}

Screening of 33 accessions for rice sheath blight resistance

Early-heading accessions had a wider range of rate of tillers with lesions $(\sim 80 \%)$ than late-heading accessions ( $\sim 40 \%$; Fig. 1). Among the mid-heading accessions, the resistant WSS3 and Jarjan showed the lowest lesion level, while the susceptible Lemont was one of accessions of the highest level. Among the early-heading accessions, Nepal 555 and Nepal 8 had the lowest levels.

\section{QTL analysis using early- to mid-heading BILs}

Earlier-heading BILs also had a wider range of rate of tillers with lesions and the heading date when the range of values dropped suddenly varied between years (Supplemental Fig. 1). So as not to score BILs which headed after this sudden drop as "resistant", we excluded those lines from the QTL analysis, assessing 63 BILs in 2009, 40 in 2010 and 35 in 2011 (Table 2).

In the three years of the study, the field tests revealed up to 11 QTLs for sheath blight resistance, on chromosomes 3, 5, 6, 9 and 12 (Table 2); however, several of these appear to represent the same QTL detected in different years. One QTL, on chromosome 9 ( $q S B R-9)$, was detected in all 3 years. Another QTL, on chromosome 5, was detected in 


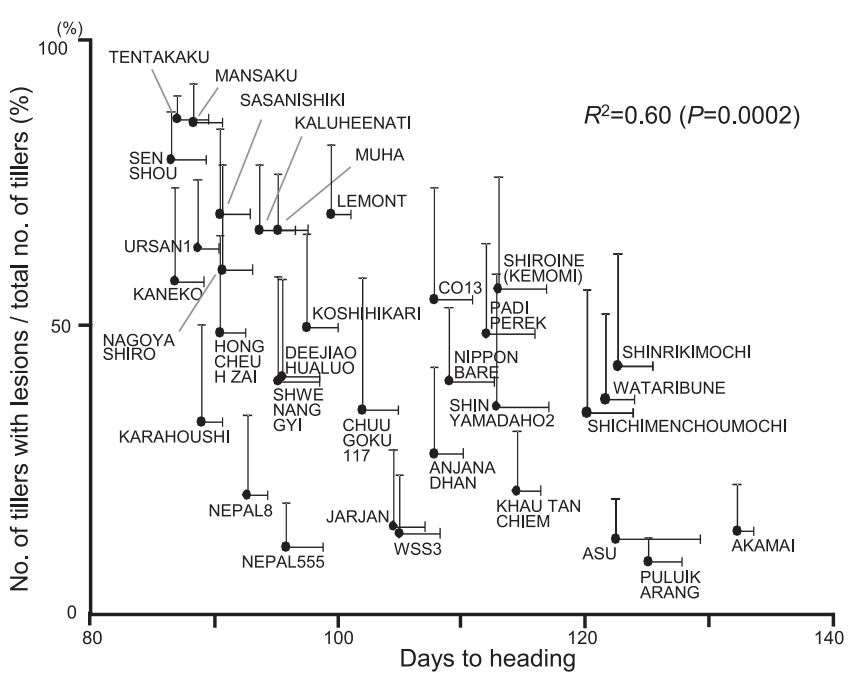

Fig. 1. Scatter plot of 33 accessions showing 3-year average days to heading and average rate of tillers with lesions. Average of two plots, each with 15 plants, per accession each year. The vertical and horizontal bars show SE.

2009 and 2011. The nearest markers to two QTLs on chromosome 3, RM570 and RM16200, were very near (at $36.5 \mathrm{Mb}$ in 2009 and $36.8 \mathrm{Mb}$ in 2010), but the QTLs were considered to be different because the directions of the addi- tive effects were opposite. Three QTLs with different locations on chromosome 6 were assumed to be different loci. Thus, we conclude that eight independent QTLs were detected across the three-year test period; for three of these ( $q S B R$ 9 [all three years], one at $36.5 \mathrm{Mb}$ on chromosome 3 [2009]) and one at $26.9 \mathrm{Mb}$ on chromosome 6 [2011]), the Jarjan allele increased resistance.

All QTLs for sheath blight resistance were identified in regions different from those of QTLs for heading date and panicle number.

\section{Validation of resistance QTL using CSSLs}

To verify $q S B R-9$, we tested four $\mathrm{BC}_{2} \mathrm{~F}_{3}(\mathrm{~J} / \mathrm{K} / / \mathrm{K})$ lines, qM3-qM6, derived from the same $\mathrm{BC}_{2} \mathrm{~F}_{2}$ plant containing a Jarjan fragment in the region of the QTL in 2010 and 2011. The SNP genotypes of the 11 SSR markers placed the Jarjan fragment between marker interval Nag08KK18184RM3855 and RM5786-1-Nag08KK18871 (Supplemental Fig. 2 left). Out of the other 151 SSR markers used to genotype the four $\mathrm{BC}_{2} \mathrm{~F}_{3}$ lines, 147 detected Koshihikari alleles. Since the heading dates of the four $\mathrm{BC}_{2} \mathrm{~F}_{3}$ lines were almost the same in each year and 10-12 days later than that of Koshihikari and the panicle numbers were similar in both years (Supplemental Fig. 2 right), we compared the rate of tillers with lesions in each line with that of the neighboring line in the field (except Koshihikari, which headed later)-

Table 2. Putative QTLs for resistance to sheath blight, number of panicles and heading date detected by using BILs (Jarjan/Koshihikari//Koshihikari)

\begin{tabular}{|c|c|c|c|c|c|c|c|c|}
\hline Year & $\begin{array}{l}\text { Number of } \\
\text { BILs used }\end{array}$ & Trait & Chr & Nearest marker & $(\mathrm{Mb})^{a}$ & $\mathrm{LOD}^{b}$ & $\mathrm{a}^{c}$ & $\mathrm{r}^{2 d}$ \\
\hline \multirow[t]{7}{*}{2009} & 63 & Rate of tillers with lesions & 3 & RM570 & 36.5 & 3.5 & 0.2 & 0.11 \\
\hline & & & 5 & RM5784 & 28.0 & 4.3 & -0.1 & 0.15 \\
\hline & & & 6 & RM1161 & 13.8 & 7.7 & -0.2 & 0.30 \\
\hline & & & 9 & RM6251 & 19.9 & 3.1 & 0.1 & 0.10 \\
\hline & & Panicle number & 6 & RM6917 & 4.1 & 2.5 & 0.9 & 0.09 \\
\hline & & Heading date & 3 & RM1350-1 & 29.5 & 6.4 & -9.0 & 0.25 \\
\hline & & & 6 & RM5850 & 11.1 & 11.5 & 11.0 & 0.37 \\
\hline \multirow[t]{9}{*}{2010} & 40 & Rate of tillers with lesions & 3 & RM16200 & 36.8 & 5.9 & -0.3 & 0.30 \\
\hline & & & 6 & RM2615 & 6.0 & 2.9 & -0.1 & 0.12 \\
\hline & & & 9 & RM6251 & 19.9 & 5.0 & 0.1 & 0.24 \\
\hline & & & 12 & RM7025 & 26.0 & 3.2 & -0.1 & 0.14 \\
\hline & & Panicle number & 7 & RM5481-1 & 16.9 & 3.0 & 0.9 & 0.46 \\
\hline & & & 12 & RM2197 & 27.5 & 3.2 & -0.7 & 0.40 \\
\hline & & Heading date & 3 & RM1350-1 & 29.5 & 6.8 & -8.6 & 0.67 \\
\hline & & & 6 & RM5850 & 11.1 & 12.0 & 10.9 & 0.56 \\
\hline & & & 7 & RM5481-1 & 16.9 & 3.3 & -5.3 & 0.58 \\
\hline \multirow[t]{5}{*}{2011} & 35 & Rate of tillers with lesions & 5 & RM3286 & 29.7 & 3.1 & -0.2 & 0.15 \\
\hline & & & 6 & RM6395 & 26.9 & 5.8 & 0.2 & 0.36 \\
\hline & & & 9 & RM3533 & 18.6 & 3.8 & 0.1 & 0.22 \\
\hline & & Panicle number & 4 & RM5478 & 34.0 & 4.1 & -1.0 & 0.20 \\
\hline & & Heading date & 6 & RM5850 & 11.1 & 5.6 & 4.9 & 0.28 \\
\hline
\end{tabular}

${ }^{a}$ According to IRGSP build 5 .

${ }^{b}$ LOD values $>2.5$ are shown.

${ }^{c}$ Additive effect of the Koshihikari allele.

${ }^{d}$ Percentage of the total phenotypic variance explained by the marker. 
A
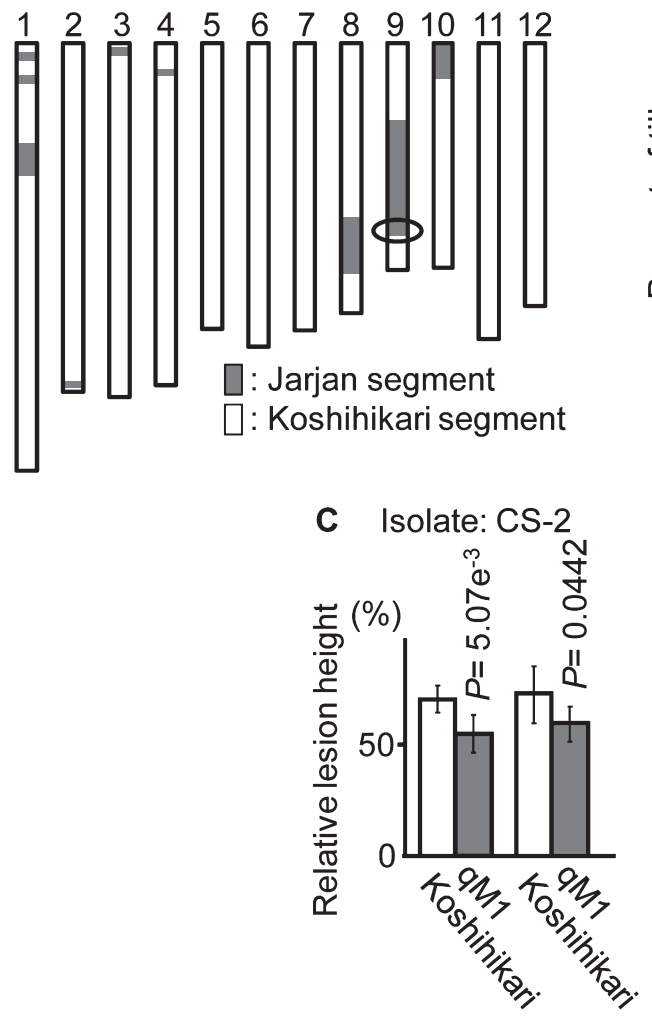

B Rhizoctonia solani isolate: 90W-14
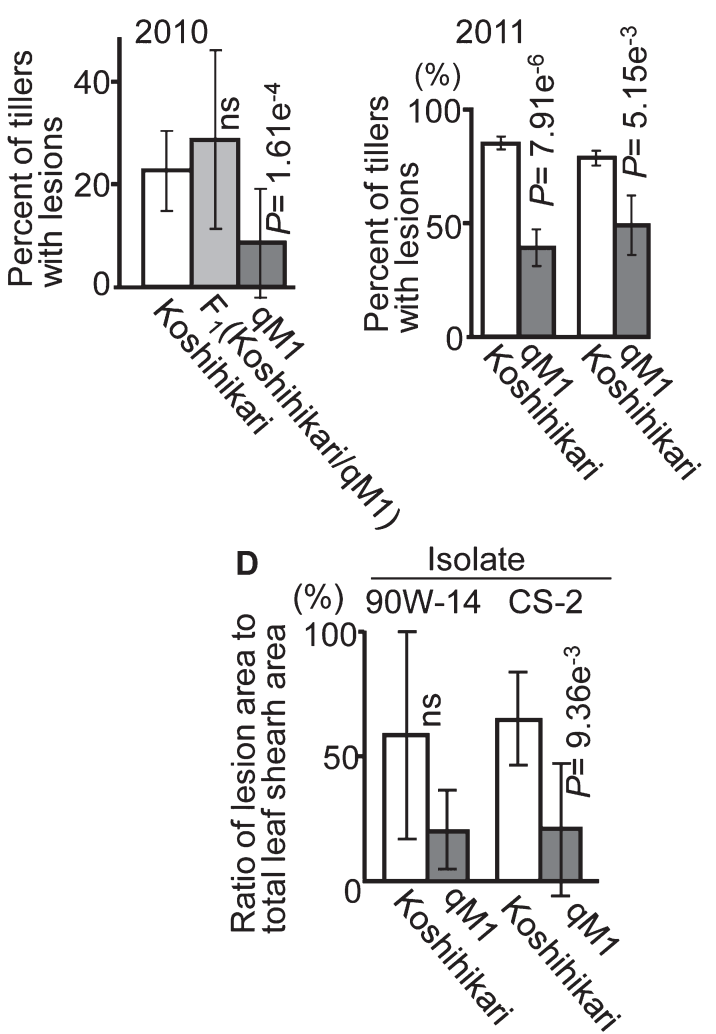

Fig. 2. (A) Graphical genotype of a $\mathrm{BC}_{2} \mathrm{~F}_{3}$ (Jarjan/Koshihikari//Koshihikari) line, qM1 and its evaluation in (B) Toyama and (C, D) Tsukuba. In each plot, heading dates of qM1 and Koshihikari differed by 1-2 days. The ellipse in (A) indicates the location of $q S B R-9$. (B, C) Soil inoculated with infected hulls. (B left) 1 plot in Toyama in 2010; (B right) 2 plots in Toyama in 2011; $n=15$. (C) 2 plots in Tsukuba in 2010; $n=5$. (D) Plants inoculated by syringe; 3 culms per plant, 5 plants per genotype.

that is, qM3 v. qM4, qM4 v. qM5 and qM5 v. qM6. qM4 was more resistant than qM3 in both years (Supplemental Fig. 2 right). qM4 and qM6 were more resistant than qM5 in 2011, however, not in 2010. Therefore, the location of the QTL could not be further narrowed down.

We compared the $\mathrm{BC}_{2} \mathrm{~F}_{3}(\mathrm{~J} / \mathrm{K} / / \mathrm{K})$ CSSL qM1, which had a Jarjan fragment in the $q S B R-9$ region, with Koshihikari because their heading dates were almost the same. In 2010, the heading date of the $\mathrm{F}_{1}(\mathrm{~K} / \mathrm{qM} 1)$ was the same as that of qM1. qM1 had Koshihikari alleles at 138 out of the 162 SSR markers. SNP typing showed that qM1 had Koshihikari alleles at 721 out of 768 SNPs (Fig. 2A). In 2010, qM1 had fewer panicles than Koshihikari, but the $\mathrm{F}_{1}$ had the same number
(Table 3). In 2011, qM1 had the same number as Koshihikari in both plots. qM1 had a lower rate of tillers with lesions than Koshihikari in 2010 and 2011 in Toyama (isolate 90W14; Fig. 2B) and in 2010 in Tsukuba (isolate CS-2; Fig. 2C), when the soil was inoculated with infected hulls. qM1 was also more resistant than Koshihikari when plants were inoculated by syringe with either strain, although because of high variability the difference in the $90 \mathrm{~W}-14$ experiment did not reach the level of significance (Fig. 2D).

$\mathrm{BC}_{3} \mathrm{~F}_{2}(\mathrm{~K} / \mathrm{qM} 1)$ lines with the Jarjan allele in the $q S B R-9$ region were more resistant than those with the Koshihikari allele in 2011 (Fig. 3).

Table 3. Panicle number of Koshihikari, $\mathrm{qM} 1\left(\mathrm{BC}_{2} \mathrm{~F}_{3}\right.$ line, $\left.\mathrm{CSSL}\right)$ and their $\mathrm{F}_{1}$

\begin{tabular}{|c|c|c|c|c|c|c|c|c|c|}
\hline \multirow[b]{3}{*}{ Cultivar or line } & \multicolumn{3}{|c|}{2010 Toyama } & \multicolumn{6}{|c|}{2011 Toyama } \\
\hline & \multirow[b]{2}{*}{ Ave } & \multirow[b]{2}{*}{ SD } & \multirow[b]{2}{*}{$P$} & \multicolumn{3}{|c|}{ Plot I } & \multicolumn{3}{|c|}{ Plot II } \\
\hline & & & & Ave & SD & $P$ & Ave & $\mathrm{SD}$ & $P$ \\
\hline Koshihikari & 15.7 & 10.4 & - & 13.6 & 2.3 & - & 12.9 & 4.6 & - \\
\hline $\mathrm{F}_{1}($ Koshihikari/qM1) & 15.1 & 10.3 & ns & nd & nd & & nd & nd & \\
\hline qM1 & 11.9 & 12.7 & $2.40 \mathrm{E}-03$ & 13.7 & 3.1 & ns & 12.6 & 1.5 & ns \\
\hline
\end{tabular}

$\mathrm{N}=15$. ns, not significant; nd, not determined. 


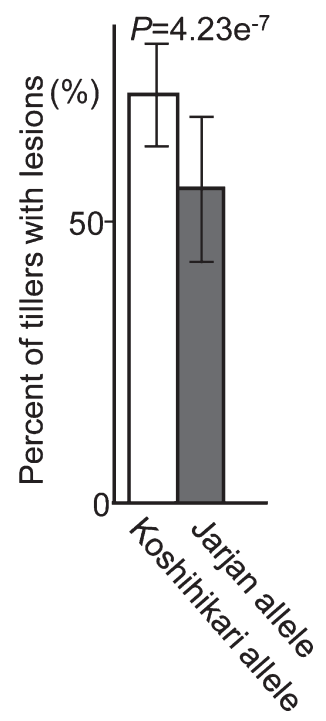

Fig. 3. Comparison of $\mathrm{BC}_{3} \mathrm{~F}_{2}$ (Koshihikari/qM1) lines with Jarjan or Koshihikari allele of $q S B R-9$ in 2011. Fifteen plants per line, 15 lines with 2 replications per genotype.

\section{Discussion}

Three years' field testing identified three landraces-Jarjan, Nepal 555 and Nepal 8-that were early to mid-heading and resistant to rice sheath blight (Fig. 1). Jarjan, a mid-heading landrace, was previously identified as resistant (Ozaki et al. 2011). All three are local landraces from the Himalayas, suggesting that some landraces from this region share genes resistant to sheath blight.

QTLs with large effects in some years could not be detected in others. Previous QTL analyses performed in multiple years or at multiple locations detected some QTLs two or three times but others only once (Han et al. 2002, Li et al. 1995, Zou et al. 2000), even when fixed lines were used for analysis. Channamallikarjuna et al. (2010) examined 127 recombinant inbred lines in seven environmental conditions at three locations across 4 years, but the QTL with the largest effect $\left(r^{2}=15 \%-26 \%\right)$ was detected in only one or two conditions. One QTL, qSBR11-1, was detected commonly in three conditions $\left(r^{2}=12 \%-14 \%\right)$, but no QTL was detected in more than three. We tested fixed lines in 3 years and likewise found that some QTLs with large effects could not be detected in every year (Table 2): only $q S B R-9$ was detected

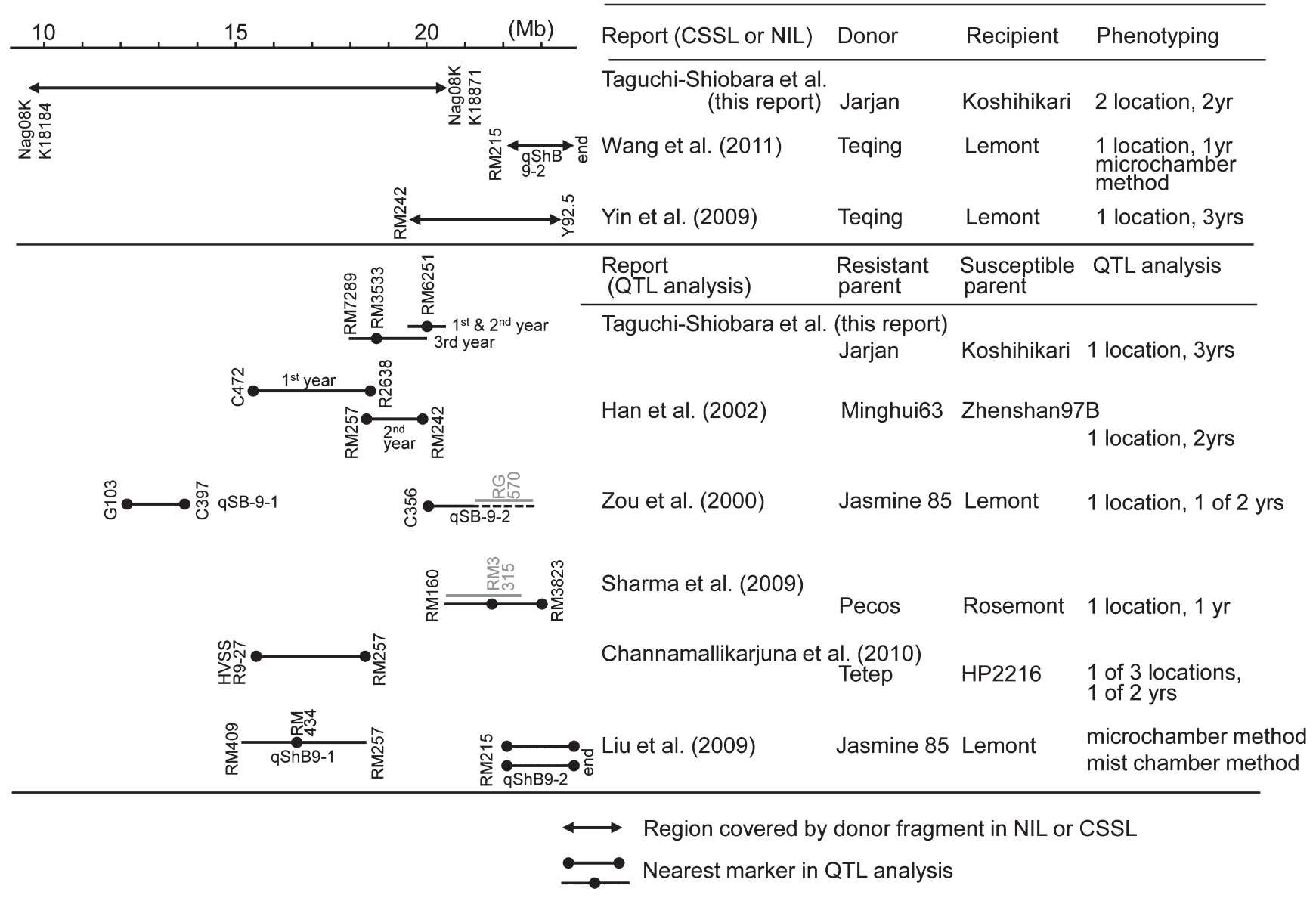

Fig. 4. Locations of QTLs for sheath blight resistance on chromosome 9 detected in this and other reports. Physical distance is according to the IRGSP Build 5 Pseudomolecules of the Rice Genome. Sequence data of two markers in gray type could not be obtained, so the locations were estimated based on the linkage map and are shown as gray horizontal bars. 
in all years, and the other seven were detected in only 1 or 2 years. In the field, genes associated with sheath blight resistance would vary by year, but those that are frequently associated with resistance would be important in breeding programs. $q S B R-9$ is a candidate because it was detected in all 3 years in Toyama, and CSSLs with a Jarjan segment in the qSBR-9 region were verified to be resistant at both Toyama and Tsukuba in 2 or more years.

We detected eight QTLs for resistance that were not associated with heading date or panicle number (Table 2). Previous studies performed QTL analysis using all lines in a hybrid population irrespective of heading date, and so scored late-heading lines as resistant without assessing the level of resistance, and coincidentally detected QTLs for resistance in the same locations as QTLs for heading date (Kunihiro et al. 2002, Li et al. 1995, Pan et al. 1999, Pinson et al. 2005, Sato et al. 2004, Sharma et al. 2009, Zou et al. 2000). In contrast, the QTLs detected here were not related to heading date or to panicle number, although the rate of tillers with lesions tends to be higher in plant with many tillers (Hashiba and Kobayashi 1996).

However, the small population size limited the detection power in this QTL analysis. Many BILs were not used each year (Table 2). Thus, increasing the number of lines in QTL analysis is important. Planting late-heading lines earlier so that they head in July (mid-summer) is one solution. Another is to use a large hybrid population that is still large enough if late-heading lines are removed. Since we did not detect the same QTLs for resistance every year, it is difficult to judge whether those detected only once or twice are genes for resistance or false positives. Creating CSSLs to test for resistance is critical.

Analysis of CSSLs containing Jarjan segments which cover the $q S B R-9$ region in the Koshihikari background confirmed the existence of the QTL. The heading date of the CSSLs was much closer to that of Koshihikari than to those of the BILs, so the levels of resistance could be compared more accurately. In contrast, four $\mathrm{BC}_{2} \mathrm{~F}_{3}$ lines (Supplemental Fig. 2) could not be compared with Koshihikari because their heading dates were 10-12 days later, and differences between them could not be clearly seen because the range of resistance became narrower in mid-August. We selected qM1, since its heading date was the same as that of Koshihikari, and verified that the Jarjan allele enhanced resistance across different locations, $R$. solani isolates and inoculation methods (Fig. 2). Furthermore, analysis of $F_{2}$ lines from a cross between Koshihikari and qM1 verified that lines carrying the Jarjan allele were more resistant than those carrying the Koshihikari allele (Fig. 3).

qSBR-9 is located in a $12.8-\mathrm{Mb}$ region between markers Nag08KK18184 (7.7 Mb) and Nag08KK18871 (20.5 Mb) (Supplemental Fig. 2 and Fig. 2A). This location overlaps that of QTLs detected in four other studies (Fig. 4 bottom). This region houses at least two resistance genes, one between 10 and $20 \mathrm{Mb}$ and the other between 22 and $24 \mathrm{Mb}$ (Fig. 4 top).
To narrow down the region in which $q S B R-9$ lies, we are currently developing recombinant inbred lines. We are developing NILs with and without the Jarjan allele of $q S B R-9$ to verify whether $q S B R-9$ can improve commercial cultivars.

\section{Acknowledgments}

We thank the field managers of NIAS and the TPAFFRC for growing the plants. We also thank Dr. S. Fukuoka for comments on the manuscript. This work was supported by grants from the Ministry of Agriculture, Forestry and Fisheries of Japan (Genomics for Agricultural Innovation, QTL-2003).

\section{Literature Cited}

Abe,T., F.Taguchi-Shiobara, Y.Kojima, T.Ebitani, M.Kuramata, T. Yamamoto, M.Yano and S.Ishikawa (2011) Detection of a QTL for accumulating $\mathrm{Cd}$ in rice that enables efficient $\mathrm{Cd}$ phytoextraction from soil. Breed. Sci. 61: 43-51.

Banniza,S. and M.Holderness (2001) Rice sheath blight-pathogen biology and diversity. In: Sreenivasaprasad, S. and R. Johnson (eds.) Major Fungal Diseases of Rice: Recent Advances, Kluwer, Dordrecht, pp. 201-211.

Channamallikarjuna, V., H. Sonah, M.Prasad, G.J.N.Rao, S.Chand, H.C.Upreti, N.K.Singh and T.R.Sharma (2010) Identification of major quantitative trait loci qSBR11-1 for sheath blight resistance in rice. Mol. Breed. 25: 155-166.

Che,K.P., Q.C.Zhan, Q.H.Xing, Z.P.Wang, D.M.Jin, D.J.He and B. Wang (2003) Tagging and mapping of rice sheath blight resistant gene. Theor. Appl. Genet. 106: 293-297.

Churchill,G.A. and R.W.Doerge (1994) Empirical threshold values for quantitative trait mapping. Genetics 138: 963-971.

Ebana,K., Y.Kojima, S.Fukuoka, T.Nagamine and M.Kawase (2008) Development of mini core collection of Japanese rice landrace. Breed. Sci. 58: 281-291.

Ebana,K., J.Yonemaru, S.Fukuoka, H.Iwata, H.Kanamori, N.Namiki, H.Nagasaki and M.Yano (2010) Genetic structure revealed by a whole-genome single-nucleotide polymorphism survey of diverse accessions of cultivated Asian rice (Oryza sativa L.). Breed. Sci. 60: 390-397.

Gangopadyay, S. and N.K.Chakrabarti (1982) Sheath blight on rice. Rev. Plant Pathol. 61: 451-460.

Gaskill,J.O. (1968) Breeding for Rhizoctonia resistance in sugarbeet. J. Am. Soc. Sugar Beet Technol. (J. Sugar Beet Res.) 15: 107-119.

Han,Y.-P., Y.-Z.Xing, Z.-X.Chen, S.-L.Gu, X.-B.Pan, X.L.Chen and Q.-F.Zhang (2002) Mapping QTLs for horizontal resistance to sheath blight in an elite rice restorer line, Minghui 63. Acta Genet. Sinica 29: 622-626.

Hashiba, T. and T.Kobayashi (1996) Rice diseases incited by Rhizoctonia species. In: Sneh,B., S.Jabaji-Hare, S.Neate and G. Dijst (eds.) Rhizoctonia Species: Taxonomy, Molecular Biology, Ecology, Pathology and Disease Control, Kluwer, Dordrecht, pp. 331-340.

Kojima,Y., K.Ebana, S.Fukuoka, T.Nagamine and M.Kawase (2005) Development of an RFLP-based rice diversity research set of germplasm. Breed. Sci. 55: 431-440.

Kunihiro, Y., Q.Qian, H.Sato, S.Teng, D.-L.Zeng, K.Fujimoto and L.-H.Zhu (2002) QTL analysis of sheath blight resistance in rice (Oryza sativa L.) Yi Chuan Xue Bao 29: 50-55.

Lander, E.S., P.Green, J.Abrahamson, A. Barlow, M.J.Daly, S.E. 
Lincoln and L.Newburg (1987) MAPMAKER: an interactive computer package for constructing primary genetic linkage maps of experimental and natural populations. Genomics 1: 174-181.

Lee,F.N. and M.C.Rush (1983) Rice sheath blight: a major rice disease. Plant Dis. 67: 829-832.

Li,Z., S.R.M.Pinson, M.A. Marchetti, J.W.Stansel and W.D. Park (1995) Characterization of quantitative trait loci (QTLs) in cultivated rice contributing to field resistance to sheath blight (Rhizoctonia solani). Theor. Appl. Genet. 91: 382-388.

Liu, G., Y.Jia, F.J.Correa-Victoria, G.A.Prado, K.M. Yeater, A. McClung and J.C.Correll (2009) Mapping quantitative trait loci responsible for resistance to sheath blight in rice. Phytopathology 99: 1078-1084.

Naito, S., T. Yamaguchi, T.Sugimoto and Y.Homma (1993) A simple method for the long-time culture storage. Annu. Rep. Plant Prot. N. Jpn. 44: 20-23.

Ohuchida,M., Y.Komaki, H.Kuwahara and T.Shigemizu (2008) Varietal differences in the resistance to sheath blight of rice. Rep. Kyushu Br. Crop Sci. Soc. Jpn. 74: 6-8.

Ozaki,H., Y.Kojima and T.Ebitani (2011) Evaluation of the varietal difference in resistance to sheath blight of rice using a cutting leaf sheath inoculum method. Hokuriku Crop Sci. 46: 18-22.

Pan, X., J.Zou, Z.Chen, J.Lu, H.Yu, H.Li, Z.Wang, X.Pan, M.C.Rush and L.Zhu (1999) Tagging major quantitative trait loci for sheath blight resistance in a rice variety, Jasmine 85 . Chinese Science Bulletin 44: 1783-1789.

Pinson, S.R.M., F.M.Capdevielle and J.H.Oard (2005) Confirming QTLs and finding additional loci conditioning sheath blight resistance in rice using recombinant inbred lines. Crop Sci. 45: 503510.

Rice Annotation Project, T.Tanaka, B.A.Antonio, S.Kikuchi, T. Matsumoto, Y.Nagamura, H.Numa, H.Sakai, J.Wu and T.Itoh et al. (2008) The Rice Annotation Project Database (RAP-DB): 2008 update. Nucleic Acids Res. 36 (Database issue): D1028-1033.

Rush,M.C. and F.N.Lee (1992) Sheath blight. In: Webster,R.K. and P.S.Gunnell (eds.) Compendium of Rice Diseases, APS Press, Minnesota, pp. 22-23.
Sato,H., I.O.Ideta, I.Ando, Y.Kunihiro, H.Hirabayashi, M.Iwano, A.Miyasaka, H.Nemoto and T.Imbe (2004) Mapping QTLs for sheath blight resistance in the rice line WSS2. Breed. Sci. 54: 265271.

Sharma,A., A.M.McClung, S.R.M.Pinson, J.L.Kepiro, A.R.Shank, R.E.Tabien and R.Fjellstrom (2009) Genetic mapping of sheath blight resistance QTLs within tropical japonica rice cultivars. Crop Sci. 49: 256-264.

Srinivasachary, L., L. Willocquet and S. Savary (2011) Resistance to rice sheath blight (Rhizoctonia solani Kuhn) [teleomorph: Thanatephorus cucumeris (A. B. Frank) Donk.] disease: current status and perspectives. Euphytica 178: 1-22.

Taguchi-Shiobara,F., Y.Kojima, T.Ebitani, M. Yano and K.Ebana (2011) Variation in domesticated rice inflorescence architecture revealed by principal component analysis and quantitative trait locus analysis. Breed. Sci. 61: 52-60.

Wang,Y., S.R.M.Pinson, R.G.Fjellstrom and R.E.Tabien (2011) Phenotypic gain from introgression of two QTL, qSB9-2 and qSB12-1, for rice sheath blight resistance. Mol. Breed. 30: 293-303.

Wasano,K. (1988) The resistance to the rice diseases of bacterial leaf blight (Xanthomonas campestris pv. oryzae) and sheath blight (Rhizoctonia solani), and some problems in breeding of disease resistance of crops. In: Japanese Society of Breeding (eds.) Recent Advances in Research on Plant Breeding vol. 30, Yokendo, Tokyo, pp. 103-121.

Wasano,K., S.Oro and Y.Kido (1983) The syringe inoculation method for selecting rice plants resistant to sheath blight, Rhizoctonia solani Kuhn. J. Trop. Agric. 27: 131-139.

Yin, Y., S.Zuo, H.Wang, Z.Cheng, S.Gu, Y.Zhang and X.Pan (2009) Evaluation of the effect of qSB-9 ${ }^{\mathrm{Tq}}$ involved in quantitative resistance to rice sheath blight using near-isogenic lines. Can. J. Plant Sci. 89: 731-737.

Zou,J.H., X.B.Pan, Z.X.Chen, J.Y.Xu, J.F.Lu, W.X.Zhai and L.H.Zhu (2000) Mapping quantitative trait loci controlling sheath blight resistance in two rice cultivars (Oryza sativa L.) Theor. Appl. Genet. 101: 569-573. 